\title{
Geometric Error Measurement of Machine Tools Using Autotracking Laser Interferometer
}

\author{
Jr-Rung Chen, ${ }^{1 *}$ Bing-Lin Ho, ${ }^{1}$ Hau-Wei Lee, ${ }^{2}$ \\ Shan-Peng Pan, ${ }^{1}$ and Tsung-Han Hsieh ${ }^{1}$ \\ ${ }^{1}$ Center for Measurement Standards, ITRI, Hsinchu City 300, Taiwan \\ ${ }^{2}$ Department of Mechanical Engineering, National Chung-Hsing University, Taizhong City 402, Taiwan
}

(Received January 18, 2018; accepted July 17, 2018)

Keywords: autotracking laser interferometer, coordinate measuring machines, machine tool, squareness errors

Geometric error measurements with traditional laser interferometers are generally complex, especially for measuring squareness errors, which require a $90^{\circ}$ pentagonal prism. Developments in the aviation industry have made the machine tools larger and distances farther. These characteristics make the optical alignment of the measurement setup difficult. An autotracking laser interferometer (ATLI) can be used to assist engineers in shortening the inspection of time machine tools by approximately $50 \%$. However, owing to the tracking function, the ATLI measuring result has minimal difference from the traditional laser interferometer. This uniquely designed interferometer is proposed in this paper for the geometric error measurement of machine tools or coordinate measuring machines (CMMs). This procedure involves the measurement of one line of an axis, and the measurement results provide information regarding the positioning and squareness errors. The experimental and simulation results show that when the linear positioning and squareness errors are generated simultaneously, the deviation first decreases then subsequently increases with the increase in test length. Hence, when the positioning error results, using ATLI, are applied to the compensation table of the controller in the machine tool, the engineer should check the result to determine whether the squareness is included too much to lead a not good compensation result.

\section{Introduction}

Machine tools and coordinate measuring machines (CMMs) with 3-5 axes have played fundamental roles in industry development. As seen in Fig. 1, a total of 21 geometric errors affect the volumetric accuracy of three-dimensional machine tools and CMMs. ${ }^{(1)}$ These errors include translation, rotation, and squareness errors. Calibrating and compensating for these geometric errors are necessary to improve the positioning accuracy of machine tools. When considering the mechanical accuracy of coordinate measuring devices, three primary sources of quasi-static errors can be identified. Geometric errors are due to the limited accuracy of

*Corresponding author: e-mail: JrRungChen@itri.org.tw https://doi.org/10.18494/SAM.2018.1979 


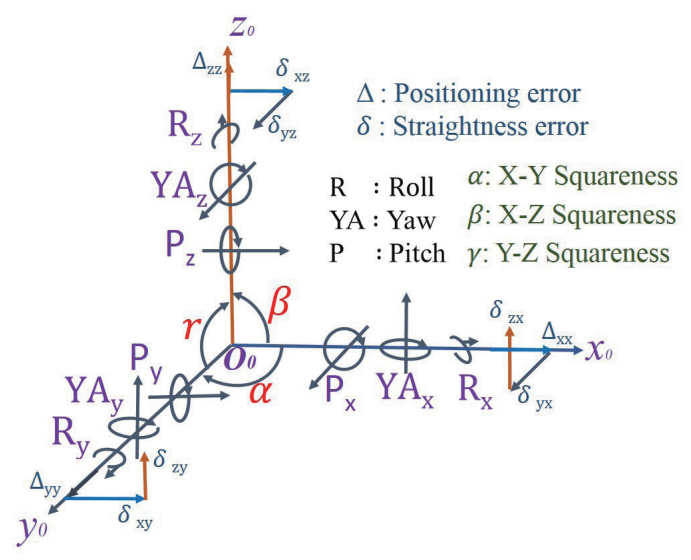

Fig. 1. (Color online) 21 geometric error terms in three-axis motion.

individual machine components, such as guideways and measuring systems. The errors related to the final stiffness of these components are mainly from the moving parts. Thermal errors are the result of the expansion and bending of guideways owing to temperature gradients.

Geometric errors are caused by straightness errors of the guideways, ${ }^{(2)}$ the imperfect alignment of the axis, and flatness errors of the manufactured surfaces. In particular, the squareness error of machine tools may arise owing to installation, shipping, or heavy cutting. The squareness error between linear motions of two axes can be estimated using diagonal displacement (ISO 230-6) tests. The linear positioning accuracy and the repeatability of the $x$-, $y$-, and $z$-axes, and the $x y, x z, y z$, and $x y z$ diagonal lines can be determined according to the ISO 230-2 and 230-6 standards. ${ }^{(3,4)}$ The aforementioned test is called the seven-line test in this paper.

A laser interferometer and an autotracking laser interferometer (ATLI) can be used to perform the seven-line test. However, the time consumed is approximately three times that of using an ATLI when using a laser interferometer. The laser tracker ${ }^{(5,6)}$ and LaserTRACER (LT) ${ }^{(7)}$ are both of the ATLI type. Lee et al. ${ }^{(8)}$ have discussed the relationship between ISO 230-2/-6 test results and the positioning accuracy of machine tools using LT. In this paper, we will further discuss the trend of the measurement result when only one ATLI is used for the squareness measurement of machine tools or CMMs.

\section{Method}

\subsection{Test procedure}

The first step in using one ATLI, following ISO 230-2 and 230-6, is fixing the ATLI onto the machine tool carriage. ${ }^{(8)}$ The next step is determining the ATLI's location related to the home/reference point of the machine tool. The measurement points for each test line are computed when the ATLI's location is determined. Numerical control (NC) codes are also generated. Users import these NC codes into the machine tool controller. The seven-line test is then performed. Subsequently, a test report can be generated after analyzing the test data. 


\subsection{ATLI coordinate determination}

The second step in the ISO 230-2 and 230-6 test procedures, as mentioned in Sect. 2.1, is determining the ATLI's coordinates on the machine tool, which can be calculated using a sixpoint measurement and the following formula:

$$
\left[\begin{array}{c}
x_{1} \\
y_{1} \\
z_{1} \\
L_{0} \\
\xi_{1}
\end{array}\right]=\left[\begin{array}{lllll}
2 x_{1} & 2 y_{1} & 2 z_{1} & 2 \Delta L_{1}-1 \\
2 x_{2} & 2 y_{2} & 2 z_{2} & 2 \Delta L_{2} & -1 \\
2 x_{3} & 2 y_{3} & 2 z_{3} & 2 \Delta L_{3} & -1 \\
2 x_{4} & 2 y_{4} & 2 z_{4} & 2 \Delta L_{4}-1 \\
2 x_{5} & 2 y_{5} & 2 z_{5} & 2 \Delta L_{5}-1 \\
2 x_{6} & 2 y_{6} & 2 z_{6} & 2 \Delta L_{6} & -1
\end{array}\right]^{\mathrm{T}}\left[\begin{array}{l}
x_{1}^{2}+y_{1}^{2}+z_{1}^{2}-\Delta L_{1}^{2} \\
x_{2}^{2}+y_{2}^{2}+z_{2}^{2}-\Delta L_{2}^{2} \\
x_{3}^{2}+y_{3}^{2}+z_{3}^{2}-\Delta L_{3}^{2} \\
x_{4}^{2}+y_{4}^{2}+z_{4}^{2}-\Delta L_{4}^{2} \\
x_{5}^{2}+y_{5}^{2}+z_{5}^{2}-\Delta L_{5}^{2} \\
x_{6}^{2}+y_{6}^{2}+z_{6}^{2}-\Delta L_{6}^{2}
\end{array}\right],
$$

where the residual measurement errors are $\zeta=x_{t}{ }^{2}+y_{t}{ }^{2}+z_{t}{ }^{2}-L_{0}{ }^{2}$ and the "+" symbol represents the pseudo-inverse operator. The six stop points $(p=1,2, \ldots, 6)$ of the cat's eye reflector are $\left(x_{p}, y_{p}, z_{p}\right)$. These points are independent and should be provided. $\left(x_{t}, y_{t}, z_{t}\right)$ are the LT coordinates to be determined. $L_{0}$ is the initial distance from the LT to the cat's eye reflector, which is unknown. $\Delta L_{i}$ is the measured distance deviation from the LT.

\subsection{ATLI measurement principle and the effect of positioning and angular error}

The measurement principle behind the applied ATLIs uses only length information. The motion of the a three-axis machine tool comprising 21 error terms results in the ideal and actual distance differences of the ATLI's coordinate determination. Figure 2 illustrates how the influence of ISO 230-2/-6 tests on $x$-axis motion with squareness error is estimated.

In Fig. 2, the solid horizontal and vertical lines represent the $x$ - and $y$-axes of the machine tool, respectively. The dotted lines represent the variation in the straightness deviation of $\theta_{x}$ in $x$-axis motion (i.e., its trajectories), which is recorded along the axis length. $\Delta x, \Delta y$, and $\Delta z$ are the shift distances between the actual and estimated location coordinates of the LT determined from Eq. (1). The measurement points are $\left(x_{i}, y_{i}, z_{i}\right)$ at different times, $i$. The length measured

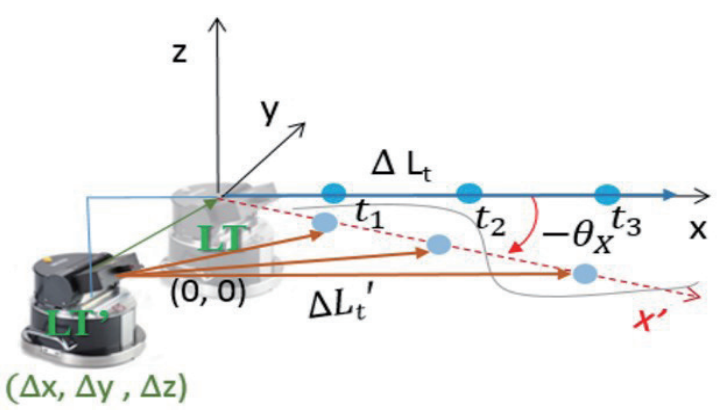

Fig. 2. (Color online) Influence of ISO 230-2/-6 test results on squareness of machine tools using an ATLI. 
by the LT is $L_{t}$. As shown in Eqs. (2) and (3), $\Delta L_{i+1}$ and $\Delta L_{i+1}^{\prime}$ indicate the ideal and actual distance differences, respectively, at the durations of $t_{i}$ and $t_{i+1}$.

$$
\begin{gathered}
\Delta L_{i+1, i}=\sqrt{\left(x_{i+1}^{2}+y_{i+1}^{2}+z_{i+1}^{2}\right)}-\sqrt{\left(x_{i}^{2}+y_{i}^{2}+z_{i}^{2}\right)} \\
\Delta L_{i+1, i}^{\prime}=\sqrt{\left(x_{i+1}^{\prime 2}+y_{i+1}^{\prime 2}+z_{i+1}^{\prime 2}\right)}-\sqrt{\left(x_{i}^{\prime 2}+y_{i}^{\prime 2}+z_{i}^{\prime 2}\right)}
\end{gathered}
$$

Here, we consider a gantry structure. The rigid-body kinematic model of the gantry structure is shown in Fig. 3. This gantry is constructed from a moving table as the $x$-axis and a fixed gantry that represents the $y$ - and $z$-axes.

$\mathrm{O}_{0}$ is the home position of the machine, $\mathrm{O}_{1}$ is the position of the target, and $\mathrm{O}_{t}$ is the location of the autotracking ranging system, which represents the center of the reference ball inside the instrument. $L_{0}+\Delta L$ is the measured distance between the ranging instrument and the moving target because, here, the incremental interferometer is considered.

Let $\left[\begin{array}{lll}s_{x} & S_{y} & s_{z}\end{array}\right]^{\mathrm{T}}$ be the idea position and $L_{V}$ be the measuring line along the distance in the $V$-axis; then, the simulated position $\left[\hat{s}_{x} \hat{s}_{y} \hat{s}_{z}\right]^{\mathrm{T}}$ with errors can be described as

$$
\left[\begin{array}{l}
\hat{s}_{x} \\
\hat{s}_{y} \\
\hat{s}_{z}
\end{array}\right]=\left[\begin{array}{c}
s_{x}+\delta_{x x} \\
s_{y}+\delta_{y x} \\
s_{z}+\delta_{z x}
\end{array}\right] .
$$

By considering a coordinate position shift $\left[\varepsilon_{x} \varepsilon_{y} \varepsilon_{z}\right]^{\mathrm{T}}$ and scale errors $g_{x}$ of position errors, the movement can be described as

$$
\left[\begin{array}{c}
\hat{s}_{x} \\
\hat{s}_{y} \\
\hat{s}_{z}
\end{array}\right]=\left[\begin{array}{c}
g_{x} s_{x}+\varepsilon_{x}+g_{x} \delta_{x x} \\
s_{y}+\varepsilon_{y}+\delta_{y x} \\
s_{z}+\varepsilon_{z}+\delta_{z x}
\end{array}\right] .
$$

Finally, the effects of positioning and angular error can be described as

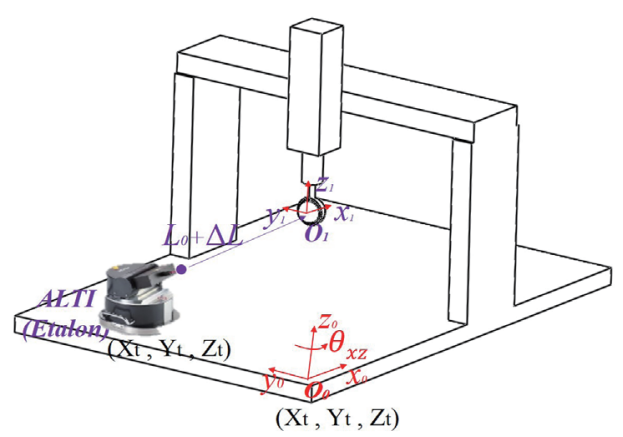

Fig. 3. (Color online) CMM appearance and each coordinate frame of the structure. 


$$
\left[\begin{array}{l}
\delta_{x x} \\
\delta_{y x} \\
\delta_{z x}
\end{array}\right]=\left[\begin{array}{ccc}
\cos \beta_{x} & 0 & \sin \beta_{x} \\
0 & 1 & 0 \\
-\sin \beta_{x} & 0 & \cos \beta_{x}
\end{array}\right]\left[\begin{array}{ccc}
\cos \gamma_{x} & -\sin \gamma_{x} & 0 \\
\sin \gamma_{x} & \cos \gamma_{x} & 0 \\
0 & 0 & 1
\end{array}\right]\left[\begin{array}{c}
s_{x} \\
0 \\
0
\end{array}\right]-\left[\begin{array}{c}
s_{x} \\
0 \\
0
\end{array}\right]
$$

Here, $\gamma_{x}$ is the squareness angle of the $x-y$ axis and $\beta_{x}$ is the squareness angle of the $x-z$ axis.

\section{Experimental Results}

The experimental setup was based on a three-axis CMM (Leitz PMM-C) in the National Measurement Laboratory in Taiwan, which is a gantry structure with an $x$-axis of $1400 \mathrm{~mm}$, a $y$-axis of $700 \mathrm{~mm}$, and a $z$-axis of $600 \mathrm{~mm}$. The simulation software was developed in VB.NET. Considering Eqs. (2) and (3), we set the squareness error of the $x-y$ axis to $0.1^{\circ}$, which is a common scale for squareness in machine tools or CMM assembly lines. The simulated and experimental results are plotted in Fig. 4. When the linear positioning and squareness errors are simultaneously generated, the deviation first decreases and subsequently increases with increasing test length. The plotted line shows an exponential curve because a squareness error will lead to a misalignment error between the ATLI location and the measurement path. Furthermore, we considered the deviation between the actual and estimated ATLI location coordinates in Eqs. (2) and (3). Here, the $x$-axis positioning error was set to $0.03 \mathrm{~mm} / \mathrm{m}$ and the squareness of the $x-y$ axis was set to $0.2^{\circ}$. As seen in Fig. 5, the resulting curve is U-shaped owing to the squareness and positioning errors. We also observed an interesting phenomenon. The curve of the measured result was in the range of -20 to $-34 \mu \mathrm{m}$. However, the estimated accuracy was only $13 \mu \mathrm{m}$. This significant offset was due to the deviation between the actual and estimated ATLI location coordinates.

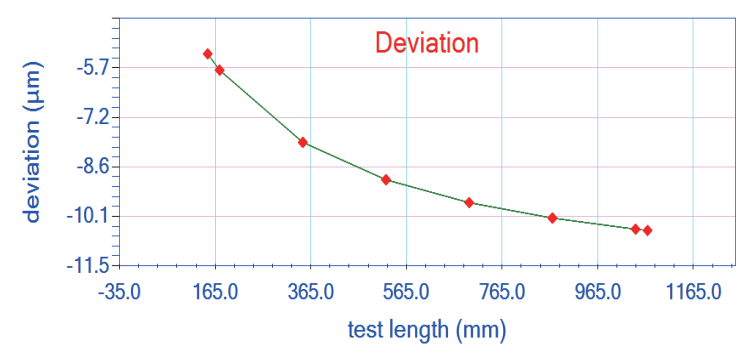

(a)

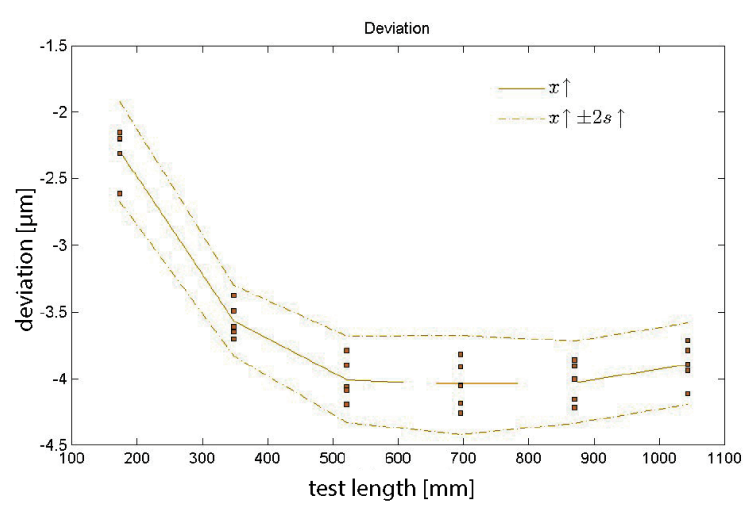

(b)

Fig. 4. (Color) (a) Simulated and (b) measured results when squareness is considered. 


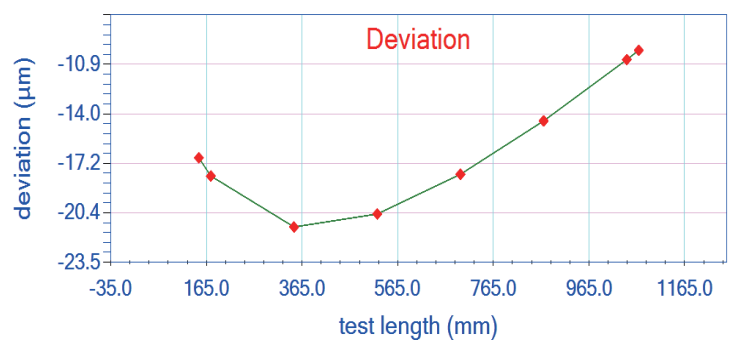

(a)

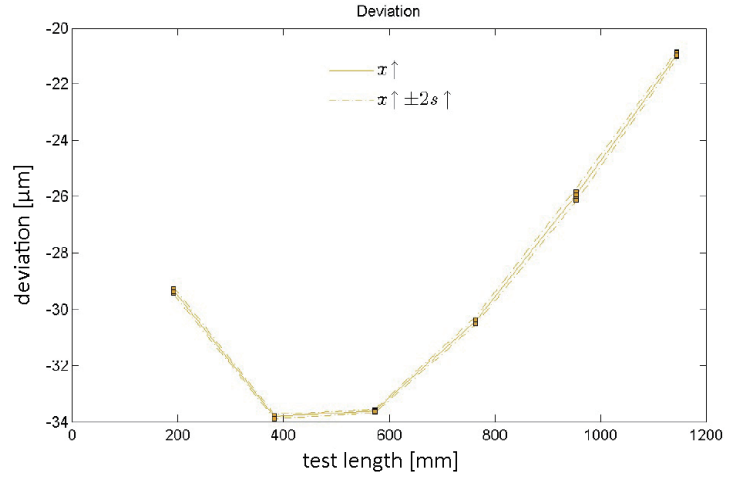

(b)

Fig. 5. (Color) (a) Simulated and (b) measured results when squareness and positioning errors are considered.

\section{Conclusions}

Compared with traditional interferometers, autotracking eases the optic alignment setup, especially for the diagonal line measurements of a machine tool. In this study, we focused on simulating the measurement results of an autotracking ranging system and provided comments on trends in the deviation results. Experimental results indicated a significant difference between positioning and squareness errors when only one axis was measured using an ATLI. A squareness error will lead to a misalignment error between the ATLI location and the measurement path. From the plotted trends in the simulated and measured results, we separate and quantify linear and squareness errors, ensuring that the use of ATLIs to perform machine tool health checks will increase ease and convenience.

\section{References}

1 G. Zhang, R. Ouyang, B. Lu, R. Hocken, R. Veale, and A. Donmez: CIRP Ann. Manuf. Technol. 37 (1988) 1.

2 W. Ptaszyñski, A. Gessner, P. Frąckowiak, and R. Staniek: Metalurgija 50 (2011) 4.

3 ISO 230: Test Code for Machine Tools, in Part 6: Determination of Positioning Accuracy on Body and Face Diagonals (ISO, 2002).

4 ISO 230: Test Code for Machine Tools, in Part 2: Determination of Accuracy and Repeatability of Positioning Numerically Controlled Axes (ISO, 2012).

5 K. Lau, R. J. Hocken, and W. C. Haight: Precis. Eng. 8 (1986) 1.

6 B. Muralikrishnan, S. Phillips, and D. Sawyer: Precis. Eng. 44 (2016).

7 PTB: Laser interferometer for spacing measurement has reference sphere positioned on post and base plate made of thermally invariant material (TRACER), German Patent No. 202004007647U1 (2004).

8 H.-W. Lee, J.-R. Chen, S.-P. Pan, H.-C. Liou, and P.-E. Hsu: Appl. Sci. 6 (2016) 4. 


\section{About the Authors}

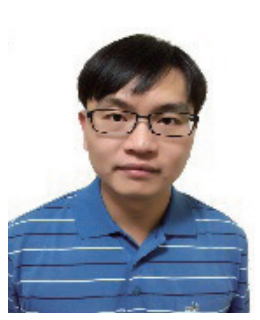

Jr-Rung Chen completed his Ph.D. at National Taiwan University of Science and Technology, Taiwan, Republic of China, in 2010. He is working at the Center for Measurement Standards, ITRI, Taiwan. His major interests are dimension metrology, precision engineering, and mechatronics.

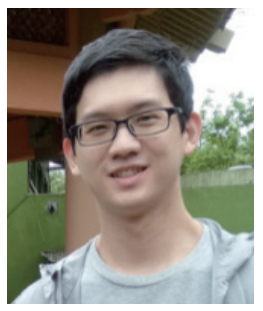

Bing-Lin Ho received his M.S. degree in 2016 from the Department of Mechanical Engineering, National Cheng-Kung University, Taiwan, Republic of China. He is a deputy engineer at the Center for Measurement Standards, ITRI, Taiwan. His main research fields are kinematic analysis, robotic surgery, machine vision, dimension metrology, and MR-compatible design.

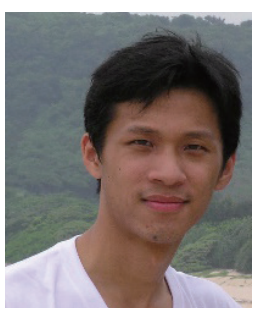

Hau-Wei Lee received his Ph.D. degree from the Institute of Aeronautics and Astronautics, National Cheng-Kung University, Taiwan, Republic of China, in 2010. From 2014 to 2017, he was a researcher at the Center for Measurement Standards, ITRI, Taiwan. He is now a researcher at the Department of Mechanical Engineering, NCHU, Taiwan. His main research fields are precision instrument design, dimension metrology, calibration of machine tools, and optomechatronic integration engineering.

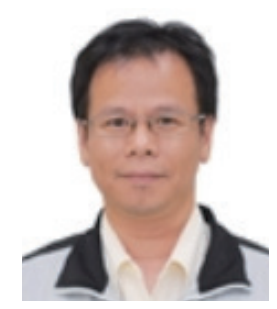

Shan-Peng Pan completed his Ph.D. degree at National Chiao Tung University, Taiwan, Republic of China, in 2010. He is working at the Center for Measurement Standards, ITRI, Taiwan. His major interests are dimension metrology and precision engineering.

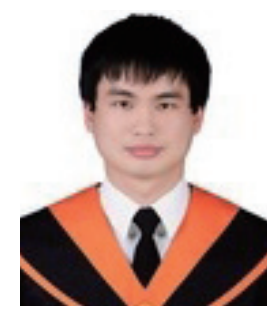

Tsung-Han Hsieh received his M.S degree from the Department of Precision Engineering, National Chung-Hsing University, Taiwan, Republic of China. $\mathrm{He}$ is a deputy engineer at the Center for Measurement Standards, ITRI, Taiwan. His research interests include dimension metrology, laser interferometry, and vision metrology. 